\title{
Photometric observations of asteroid 4 Vesta by the OSIRIS cameras onboard the Rosetta spacecraft ${ }^{\star}$
}

\author{
S. Fornasier ${ }^{1,2}$, S. Mottola ${ }^{3}$, M. A. Barucci ${ }^{1}$, H. Sierks ${ }^{4}$, and S. Hviid ${ }^{4}$ \\ 1 LESIA-Observatoire de Paris, CNRS, UPMC Univ. Paris 06, Univ. Paris-Diderot, 5 place J. Janssen, 92195 Meudon Pricipal Cedex, \\ France \\ e-mail: sonia.fornasier@obspm.fr \\ 2 Univ. Paris Diderot, Sorbonne Paris Cité, 4 rue Elsa Morante, 75205 Paris, France \\ 3 Institute of Planetary Research, DLR, Rutherfordstrasse 2, 12489 Berlin, Germany \\ ${ }^{4}$ Max-Planck-Institut für Sonnensystemforschung, Max-Planck-Strasse 2, 37191 Katlenburg-Lindau, Germany \\ Received 30 June 2011 / Accepted 13 August 2011
}

\section{ABSTRACT}

\begin{abstract}
Aims. We report on new observations of asteroid 4 Vesta obtained on 1 May 2010 with the optical system OSIRIS onboard the ESA Rosetta mission. One lightcurve was taken at a phase angle $\left(52^{\circ}\right)$ larger than achievable from ground-based observations together with a spectrophotometric sequence covering the 260 to $990 \mathrm{~nm}$ wavelength range.

Methods. Aperture photometry was used to derive the Vesta flux at several wavelengths. A Fourier analysis and the HG system formalism were applied to derive the Vesta rotational period and characterize its phase function.

Results. We find a G parameter value of $0.27 \pm 0.01$ and an absolute magnitude $H(R)=2.80 \pm 0.01$. The lightcurve has the largest amplitude ever reported for Vesta $(0.19 \pm 0.01 \mathrm{mag})$, and we derive a synodic rotational period of $5.355 \pm 0.025 \mathrm{~h}$. The Rosetta spectrophotometry, covering the Vesta western hemisphere, is in perfect agreement with visible spectra from the literature and close to the IUE observations related to the same hemisphere. The new spectrophotometric data reveal that there is no global ultraviolet/visible reversal on Vesta. The Vesta spectrophotometry is well reproduced by spectra of howardite meteorite powders (grain size $<25 \mu \mathrm{m}$ ). From the Rosetta absolute spectrophotometry and from the phase function behaviour, we estimate a geometric albedo of $0.36 \pm 0.02$ at $649 \mathrm{~nm}$ and $0.34 \pm 0.02$ at $535 \mathrm{~nm}$.
\end{abstract}

Key words. minor planets, asteroids: individual: 4 Vesta - techniques: photometric

\section{Introduction}

Vesta is the most massive asteroid in the main belt (considering that Ceres has been classified as a dwarf planet) orbiting the Sun at a distance of about 2.36 AU. From HST images, Thomas et al. (1997a) derived a Vesta shape fit by an ellipsoid with semi-axes of $289,280,229( \pm 5) \mathrm{km}$, and J2000 pole coordinates RA $=308 \pm 10^{\circ}$, Dec $=48 \pm 10^{\circ}$, which was more recently updated to $\mathrm{RA}=305.8 \pm 3.1^{\circ}, \mathrm{Dec}=41.4 \pm 1.5^{\circ}(\mathrm{Li}$ et al. 2011a). These HST data were also analysed to uncover a large (460 km wide) basin near the south pole with a pronounced central peak of $13 \mathrm{~km}$, together with other depressions and large craters (Thomas et al. 1997b). The discovery of substantial impact excavation on Vesta is consistent with the idea that this asteroid is the source of HED meteorites. Several "Vestoids", i.e. asteroids with spectral properties and orbital parameters similar to Vesta, have also been discovered. They have therefore probably been caused by impact events on Vesta.

Vesta is large enough to have experienced a differentiation phase during its accretion and is the only large asteroid known to have a basaltic surface that retains a record of ancient volcanic activity (McCord et al. 1970; Gaffey et al. 1997). Geological diversity revealing longitudinal variations in albedo and mineralogy was detected from polarimetric and spectroscopic measurements obtained at different rotational phases (Gaffey 1997; Binzel et al. 1997; Li et al. 2010).

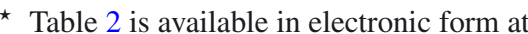
http: //www . aanda.org
Vesta is the first target of the NASA Dawn mission, that just entered orbit around the asteroid in July 2011 and will remain in orbit for one complete year, undertaking a detailed study of its geophysics, mineralogy, and geochemistry (Russell et al. 2007; Sierks et al. 2011).

In this paper we report on observations of Vesta obtained with the scientific optical camera system OSIRIS onboard Rosetta. One new lightcurve taken at an unprecedented large phase angle $\left(52^{\circ}\right)$ has been obtained together with spectrophotometry from 260 to $990 \mathrm{~nm}$. These data, taken at a nearly equatorial aspect, allow us to more tightly constrain the Vesta phase function, to provide the absolute reflectance from the UV to the NIR range at large phase angle, and to estimate the geometric albedo.

\section{Observations and data reduction}

OSIRIS is the imaging system onboard Rosetta mission. It consists of a narrow-angle camera (NAC, field of view of $2 \times 2^{\circ}$ ) and a wide-angle camera (WAC, field of view of $12 \times 12^{\circ}$ ). They are unobstructed mirror systems with focal lengths of $72 \mathrm{~cm}$ and $14 \mathrm{~cm}$, respectively. Both cameras are equipped with $2048 \times$ 2048 pixel CCD detectors with a pixel size of $13.5 \mu \mathrm{m}$. The image scale is $3.9 \mathrm{arcsec} / \mathrm{pixel}$ for the NAC and $20.5 \mathrm{arcsec} / \mathrm{pixel}$ for the WAC. The cameras have a set of broadband and narrowband filters covering the wavelength range 240-990 nm. We refer to Keller et al. (2007) for a detailed description of the instrument. 
During its approach to asteroid 21 Lutetia, which was successfully flown by on 10 July 2010, Rosetta observed the asteroid 4 Vesta on 1 May 2010 for a total duration of $10.8 \mathrm{~h}$. These observations included lightcurve coverage with the two "red" filters of the cameras (the NAC filter F22 centred at $649.2 \mathrm{~nm}$, and the WAC filter F12, centred at $629.8 \mathrm{~nm}$ ), and a spectrophotometric sequence including 12 NAC filters and 9 WAC filters, covering the wavelength range 269-989 nm (Table 1). The observations were made at a spacecraft-target distance $0.285879<$ $\Delta<0.283682 \mathrm{AU}$, at a Vesta heliocentric distance of $2.324 \mathrm{AU}$, and at a phase angle $52.1<\alpha<52.8^{\circ}$, the largest one covered up to date, before the DAWN encounter. Vesta was observed at equatorial aspect, at a sub-spacecraft latitude of $3.0-2.7^{\circ}$, and was not resolved (resolution of $800 \mathrm{~km} / \mathrm{px}$ and $4000 \mathrm{~km} / \mathrm{px}$ with the NAC and WAC cameras, respectively).

The data were reduced using the OSIRIS standard pipeline, but the flux calibration was done using revised values of the conversion factors that had been recently computed. The data reduction steps are the same as those described in Küppers et al. (2007). The data are converted from digital units to $\mathrm{W} \mathrm{m}^{-2} \mathrm{~nm}^{-1} \mathrm{sr}^{-1}$, using conversion factors derived from observations of Vega taken on 1 May 2010 and 12 July 2010. These factors were reduced to a solar input spectrum. The absolute calibration factors were computed using the Vega (alpha_lyr_stis_005.ascii) and sun flux (sun_reference_stis_001.ascii) standard spectra from the HST CALSPEC catalogue ${ }^{1}$.

The Vesta flux was calculated from the images using aperture photometry with an aperture radius of five (WAC) and six (NAC) pixels. These radii allow us to acquire more than $99 \%$ of the target flux and to minimize the background contribution and cosmic ray hits. Aperture correction factors were derived from the high signal-to-noise ratio observations of Vega and the fluxes were divided by 0.992446 for the WAC and 0.992918 for the NAC observations to get the total flux (estimated from Vega growth curves for an aperture of $15 \mathrm{px}$ ). The background was evaluated in four rectangular regions around the target and then subtracted from the total flux.

\section{Lightcurve and phase function}

We used the 44 WAC and NAC images in the red filters to build the Vesta lightcurve. To derive the absolute magnitude at the observed phase angle, we follow the method described by Küppers et al. (2007). We first corrected the Vesta flux by considering its spectrum, which is redder than that of the sun, using:

$F_{\mathrm{c}}=F_{\mathrm{o}} \times \frac{\int_{\lambda} F_{\odot}(\lambda) T(\lambda) \mathrm{d} \lambda}{\int_{\lambda} F_{\text {Vesta }}(\lambda) T(\lambda) \mathrm{d} \lambda}$,

where $F_{\mathrm{c}}$ and $F_{\mathrm{o}}$ are the corrected and uncorrected Vesta fluxes at a given central filter wavelength $\lambda_{\mathrm{c}}, T(\lambda)$ is the system throughput (telescope optics and CCD quantum efficiency), $F_{\odot}(\lambda)$ and $F_{\text {Vesta }}(\lambda)$ are solar (from HST catalogue) and Vesta spectra (from Xu et al. 1995), respectively, both normalized to unity at the $\lambda_{\mathrm{c}}$ of the considered filter.

The absolute magnitude $R(1,1, \alpha)$ reduced to the $R$-Bessel filter was finally computed, and we derived a mean value of $4.485 \pm 0.003$. The data were phase corrected to $\alpha=52.26^{\circ}$ using the HG phase function with $g=0.27$, as described later in the paper. The results are shown in Fig. 1 and reported in Table 2. The lightcurve is single peaked, consistent with an albedo driven

\footnotetext{
1 www.caha.es/pedraz/SSS/HST_CALSPEC
}

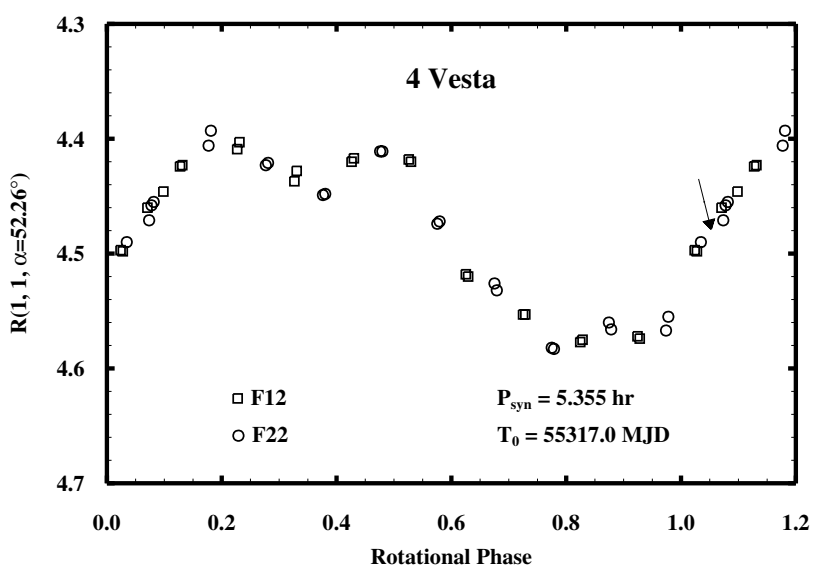

Fig. 1. The lightcurve of asteroid 4 Vesta from OSIRIS observations. The arrow show the rotational phase corresponding to the spectrophotometric data set acquisition. Points beyond rotational phase 1.0 are repeated for clarity. F12 and F22 are the red filters of the WAC and NAC cameras, respectively.

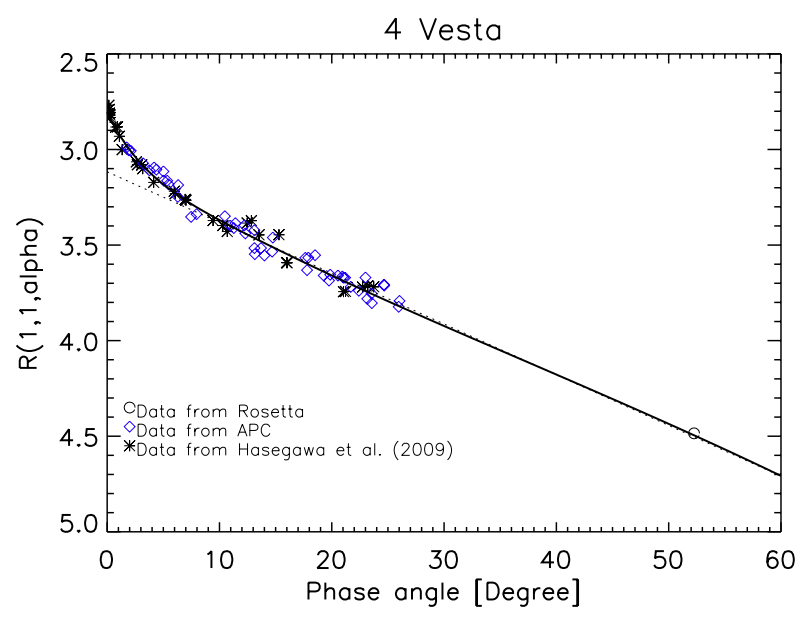

Fig. 2. Phase curve of asteroid 4 Vesta. Beside the Rosetta point, data come from the Asteroid Photometric Catalogue (Lagerkvist et al. 1995) and from Hasegawa et al. (2009). The dashed-line represents the linear fit to the data.

behaviour. Its amplitude is $0.19 \pm 0.01$, the largest one observed so far for Vesta as a consequence of the well-known amplitudephase effect (Zappalá et al. 1995).

The Vesta time-series were modelled with a ninth order Fourier polynomial (Harris et al. 1989), whose best-fit relation corresponded to a synodic rotation period $\left(P_{\text {syn }}\right)$ of $5.355 \pm$ $0.025 \mathrm{~h}$. The relatively large error is related to the limited timespan of the Rosetta observations. The data folded with the bestfit period are shown in Fig. 1. The Rosetta $P_{\text {syn }}$ is slightly longer, but still compatible within the error bars, than the very precise value of the sidereal period $(5.34212971 \pm 0.00000096 \mathrm{~h}) \mathrm{de}-$ rived by Drummond et al. (1998).

The Rosetta observations, taken at a large phase angle, are important to constrain the Vesta phase function. We compiled the asteroid phase curve in $R$ band (Fig. 2) combining the Rosetta data with those coming from the Asteroid Photometry Catalogue (APC, $V$ filter) dataset (Lagerkvist et al. 1995), and from Hasegawa et al. (2009, $R$ filter). We used a $V-R$ colour index of 0.385 , derived from a Vesta visual spectrum (Xu et al. $1995)$ to combine the visual magnitude from the APC with the other data. We take the mean value from each individual data 
Table 1. Vesta spectrophotometry from Osiris.

\begin{tabular}{lcccccc}
\hline \hline UT $_{\text {start }}$ & Inst. & $F$ & $\lambda_{\text {c }}$ & exp. $(\mathrm{s})$ & $F_{\text {Abs }}(\alpha)$ & $p$ \\
\hline $10: 56: 26$ & NAC & 15 & 269.30 & 48.52 & $0.021 \pm 0.002$ & 0.10 \\
10:57:26 & NAC & 16 & 360.00 & 1.67 & $0.042 \pm 0.002$ & 0.20 \\
10:57:39 & NAC & 28 & 743.70 & 0.26 & $0.080 \pm 0.001$ & 0.38 \\
10:57:50 & NAC & 27 & 701.20 & 0.74 & $0.078 \pm 0.002$ & 0.37 \\
10:58:03 & NAC & 24 & 480.70 & 0.23 & $0.066 \pm 0.002$ & 0.32 \\
10:58:14 & NAC & 23 & 535.70 & 0.24 & $0.071 \pm 0.001$ & 0.34 \\
$10: 58: 25$ & NAC & 22 & 649.20 & 0.15 & $0.076 \pm 0.001$ & 0.36 \\
$10: 58: 37$ & NAC & 41 & 882.10 & 0.70 & $0.057 \pm 0.001$ & 0.27 \\
$10: 58: 49$ & NAC & 51 & 805.30 & 0.79 & $0.072 \pm 0.001$ & 0.34 \\
$10: 59: 01$ & NAC & 61 & 931.90 & 1.96 & $0.055 \pm 0.001$ & 0.26 \\
$10: 59: 14$ & NAC & 71 & 989.30 & 4.34 & $0.061 \pm 0.002$ & 0.29 \\
$10: 59: 30$ & NAC & 58 & 790.50 & 10.00 & $0.075 \pm 0.001$ & 0.36 \\
$11: 00: 29$ & WAC & 18 & 612.60 & 14.70 & $0.073 \pm 0.002$ & 0.35 \\
$11: 00: 52$ & WAC & 17 & 631.60 & 57.77 & $0.075 \pm 0.003$ & 0.36 \\
$11: 01: 57$ & WAC & 16 & 590.70 & 43.57 & $0.073 \pm 0.003$ & 0.35 \\
$11: 02: 48$ & WAC & 15 & 572.10 & 16.76 & $0.072 \pm 0.002$ & 0.35 \\
$11: 03: 13$ & WAC & 14 & 388.40 & 270.88 & $0.054 \pm 0.006$ & 0.26 \\
$11: 07: 51$ & WAC & 13 & 375.60 & 119.46 & $0.048 \pm 0.004$ & 0.23 \\
$11: 09: 58$ & WAC & 12 & 629.80 & 1.00 & $0.076 \pm 0.001$ & 0.36 \\
$11: 10: 07$ & WAC & 21 & 537.20 & 2.74 & $0.072 \pm 0.001$ & 0.34 \\
$11: 10: 18$ & WAC & 71 & 325.80 & 441.86 & $0.029 \pm 0.006$ & 0.14 \\
\hline
\end{tabular}

Notes. The observations were acquired on 1 May 2010, at a phase angle of $52.45-52.47^{\circ}$ and at a Rosetta-Vesta distance of $0.284857285-0.284809694$ AU. $F$ is the filter combination, $\lambda_{c}$ the filter central wavelength, exp. the exposure time, $F_{\mathrm{Abs}}$ the absolute reflectance at $\alpha=52.4^{\circ}$, and $p$ the geometric albedo estimated assuming the same phase function for all the wavelengths.

set of observations from Hasegawa et al. (2009). The data correspond to very different Vesta aspects, which causes considerable scatter in the data (Fig. 2). The $H-G$ function (Bowell et al. 1989) that most closely fits the data gives an $H_{R}=2.80 \pm 0.01$ and $G=0.27 \pm 0.01$. Our $G$ value, well-constrained by the Rosetta point at $\alpha=52^{\circ}$, is in-between the previous estimates of Lagerkvisk et al. (1990, $G=0.33$ in $V$ filter) and Hasegawa et al. (2009, $G=0.21$ in $R$ filter). Vesta displays a high opposition effect: its linear slope (calculated for $\alpha>7^{\circ}$ ) is $0.02654 \mathrm{mag} /{ }^{\circ}$ and the magnitude from the linear slope (thereby excluding the opposition effect) is $R_{\operatorname{lin}}=3.12$. The Vesta linear slope and opposition surge values are comparable with those of S-type asteroids (Belskaya \& Shevchenko 1999). The new $H$ and $G$ values can be used to estimate the Vesta albedo (see following section) and its flux at large phase angles.

\section{Spectrophotometry}

A spectrophotometric sequence including 12 NAC filters and 9 WAC filters, covering the wavelength range 269-989 nm, was acquired at a sub-spacecraft latitude of $2.8^{\circ}$ and a sub-spacecraft longitude of $205-221^{\circ}$ (the longitude and latitude were calculated from the Rosetta spice kernels following the IAU convention, that is longitude increasing in the East direction). Rosetta looked then mostly at the western hemisphere. The details of these observations together with the absolute reflectance value at a phase angle of $52.4^{\circ}$ are reported in Table 1 . The absolute reflectance $\left(F_{\text {Abs }}\right)$ was computed for each filter as follows

$F_{\mathrm{Abs}}\left(\alpha=52.4^{\circ}\right)=\frac{F_{\mathrm{V}} \times R_{\odot}^{2} \times \Delta^{2}}{r_{\mathrm{V}}^{2} * F_{\odot}}$,

where $F_{\mathrm{V}}$ is the Vesta flux expressed in $\mathrm{W} / \mathrm{m}^{2} / \mathrm{nm}, R_{\odot}$ is the heliocentric distance of Vesta at the time of observation

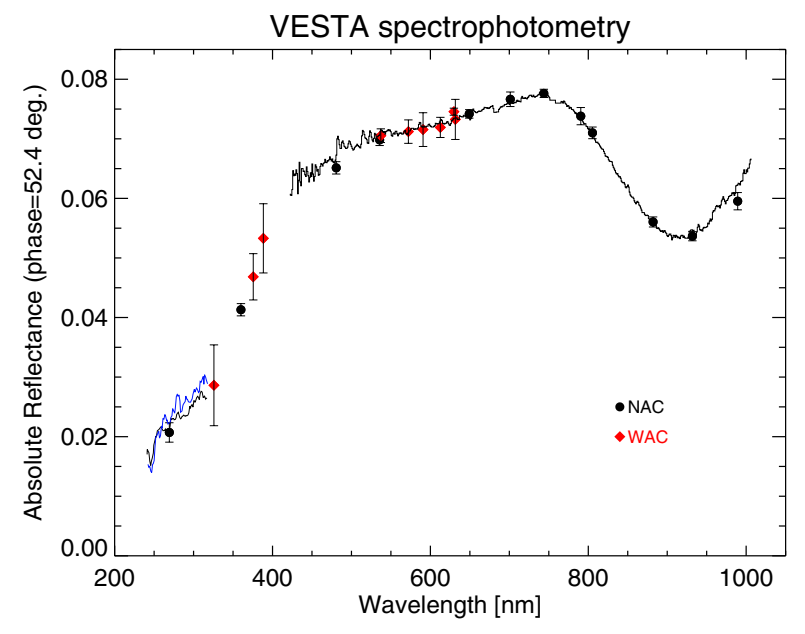

Fig. 3. Spectrophotometry of 4 Vesta from the WAC and NAC cameras of the OSIRIS imaging system. For comparison, a ground-based spectrum taken from the SMASS survey is shown (Xu et al. 1995). In the UV, data come from the IUE telescope: in blue the LWP 18955 observation, in black the LWP 18952 one (Hendrix et al. 2003).

(2.3241538 AU), $\Delta$ the distance between Rosetta and the target (in $\mathrm{AU}), r_{\mathrm{V}}$ the Vesta radius $(260 \mathrm{~km}$, estimated from the Vesta cross-section area at the Rosetta observing conditions using the Thomas et al. (1997a) shape model), and $F_{\odot}$ is the solar flux (in $\mathrm{W} / \mathrm{m}^{2} / \mathrm{nm}$ ) through a given filter.

We can estimate the geometric albedo multiplying the absolute reflectance by a phase correction factor derived from the phase function $(G=0.27)$. With this method, we find a geometric albedo of $0.36 \pm 0.02$ at $649 \mathrm{~nm}$. In Table 1, we report the geometric albedo estimated for all the Rosetta observations, assuming the same phase function for the different wavelengths. These values must be interpreted with caution for the UV and NIR regions, as we do not know the wavelength dependence of the phase curve.

Tedesco et al. (2002) reported a geometric albedo of $0.42 \pm 0.05$ at visible wavelength from IRAS data. However, they used a diameter value of $468 \mathrm{~km}$, which is smaller than that estimated from HST images (Thomas et al. 1997a). Using Thomas' et al. size, their geometric albedo would be $0.34(\mathrm{Li}$ et al. 2011b), in perfect agreement with the Rosetta one.

The Osiris spectrophotometry of Vesta is shown in Fig. 3. For comparison, we present a visual spectrum from the SMASS survey (Xu et al. 1995, spectrum resulting from the mean of three separate observations taken at sub-Earth lat. $24-38^{\circ}$ ), scaled to the Rosetta absolute reflectance at $535 \mathrm{~nm}$, and two UV spectra from IUE observations (Hendrix et al. 2003), scaled to the same phase angle of the Rosetta observations with our phase function parameters. The IUE observations correspond to the sets LWP 18952 and LWP 18955 that Hendrix et al. related to the eastern and western hemispheres, respectively. Hendrix et al. (2003) claim to have detected evidence of Vesta's spectral reversal, that is regions that are brighter in the visible range (Vesta eastern hem.) seem to be darker in the UV range. We recalculated the sub-Earth longitude $\left(\lambda_{\text {subE }}\right)$ of the IUE observations with the IMCCE ephemeris server ${ }^{2}$ finding $\lambda_{\text {subE }}=277^{\circ}$ for the LWP 18952 set, corresponding to the western hemisphere, and $\lambda_{\text {subE }}=133^{\circ}$ for the LWP 18955, corresponding to the eastern hemisphere, opposite to the Hendrix et al. (2003) results. As shown in Fig. 3, the Rosetta data,

2 http://www.imcce.fr 


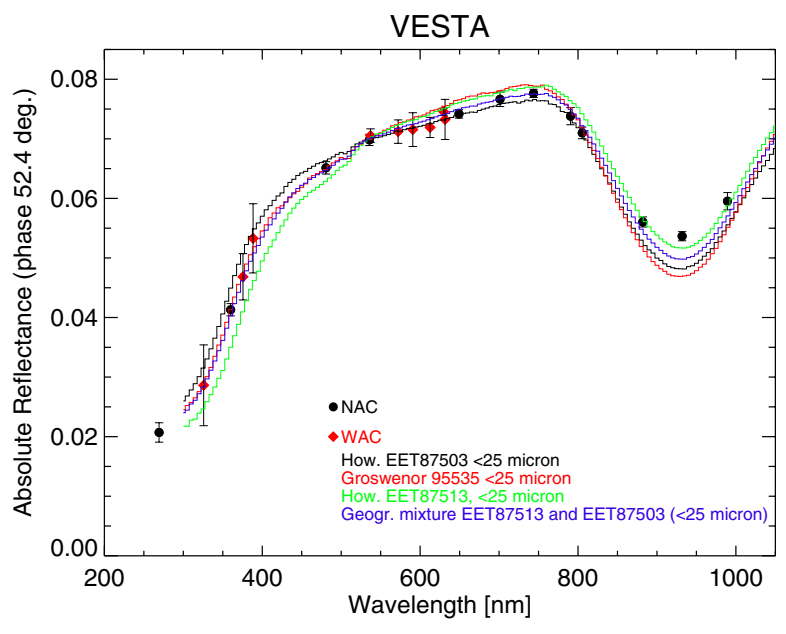

Fig. 4. Spectrophotometry of 4 Vesta from OSIRIS imaging system with the best meteorite analogues found.

taken in the western hemisphere, are in good agreement with the SMASS spectrum and close to the IUE LWP 18952 observation, which also seems to fall in the western hemisphere according to the revisited $\lambda_{\text {sube }}$. The Rosetta data, taken from UV to NIR almost simultaneously, are inconsistent with the UV spectral reversal. Analysing UV data from HST and Swift telescopes and combining them with V+NIR data, also Li et al. (2011) conclude that there is no global ultraviolet/visible reversal on Vesta, implying lack of global space weathering.

The band-pass of the NIR filters is too broad to determine a meaningful mineralogical characterisation from the absorptionband position and depth in the 0.9 micron region. We refer the reader to the works, for example, of Gaffey et al. (1997), Binzel et al. (1997), Burbine et al. (2001), and Reddy et al. (2010) for the mineralogical interpretation of Vesta. We attempt to characterize the observed Vesta spectrum by looking for meteorite analogues from the RELAB database. It is well-known that Vesta is associated with the basaltic achondrites howardite, eucrite, and diogenite (HED) (Clayton \& Mayeda 1983; McSween et al. 2011). These meteorites are the equivalent of terrestrial magmatic rocks, and their igneous appearance proves the existence of magmatic activity on their parent body very early in the evolution of the Solar System (McSween 1989). The spectra of individual HED meteorites are quite diverse in both terms of spectral slope and $0.9-1 \mu \mathrm{m}$ and $2 \mu \mathrm{m}$ absorption bands centre and depth, even for similar grain sizes (Burbine et al. 2001; Hiroi et al. 1994). This is directly linked to meteorite mineralogy as reported by Pieters et al. (2006).

We find that the Rosetta spectrophotometric data are close to the spectral behavior of fine-grained powder samples (grain size $<25 \mu \mathrm{m}$ ) of howardite meteorites (Fig. 4). A geographical mixture obtained with $50 \%$ of EET87503 and $50 \%$ of EET87513 howardites, both from Elephant Moraine (Antarctica), shows a very good match to the Rosetta data in the $260-850 \mathrm{~nm}$ region, but has a slightly larger $0.93 \mu \mathrm{m}$ depth. This mixture has an albedo of 0.36 at $535 \mathrm{~nm}$, compatible with the estimated Vesta geometric albedo. Additional components, which must also be constrained using Vesta data in the NIR range, are needed to improve the spectral match. Our meteorite match confirms the results previously found by Hiroi et al. (1994), suggesting that Vesta's surface must be covered by relatively fine-grained basaltic materials.

\section{Conclusions}

We have analysed Rosetta data of asteroid Vesta taken at a phase angle of $\sim 52^{\circ}$ to extend and more tightly constrain its phase function. Using the $H-G$ formalism, we have found a $G$ parameter value of $0.27 \pm 0.01$ and an absolute magnitude $H(R)=$ $2.80 \pm 0.01$. The new lightcurve has the largest amplitude ever seen for this object. The Rosetta absolute reflectance obtained in 19 filters covering the near UV up to the near IR region does not support the previously reported spectral reversal suggested by Hendrix et al. (2003). The Rosetta data are well-matched from the UV region up to about $850 \mathrm{~nm}$ by a mixture of two howardite fine-grained powder samples. This mixture has an albedo similar to that of Vesta but a deeper $0.93 \mu \mathrm{m}$ band.

We have estimated from our absolute reflectance at $\alpha=52^{\circ}$ and the phase function a Vesta geometric albedo of $0.36 \pm 0.02$ at $649 \mathrm{~nm}$ and $0.34 \pm 0.02$ at $535 \mathrm{~nm}$. This information will be very useful to the Dawn team in preparing their observations. These results attest to the excellent capabilities of the OSIRIS camera in terms of both scientific usefulness and technical performance.

Acknowledgements. S.F. thanks Dr. Li for helpful discussion and information exchange, and Dr. Hasegawa for proving his phase function data. OSIRIS was built by a consortium of the Max-Planck-Institut für Sonnensystemforschung, Katlenburg-Lindau, Germany, CISAS-University of Padova, Italy, the Laboratoire d'Astrophysique de Marseille, France, the Instituto de Astrofísica de Andalucia, CSIC, Granada, Spain, the Research and Scientific Support Department of the European Space Agency, Noordwijk, The Netherlands, the Instituto Nacional de Técnica Aeroespacial, Madrid, Spain, the Universidad Politéchnica de Madrid, Spain, the Department of Physics and Astronomy of Uppsala University, Sweden, and the Institut für Datentechnik und Kommunikationsnetze der Technischen Universität Braunschweig, Germany. The support of the national funding agencies of Germany (DLR), France (CNES), Italy (ASI), Spain (MEC), Sweden (SNSB), and the ESA Technical Directorate is gratefully acknowledged. This research utilizes spectra acquired with the NASA RELAB facility at Brown University.

\section{References}

Belskaya, I. N., \& Shevchenko, V. G. 1999, Icarus, 147, 94

Binzel, R. P., Gaffey, M. J., Thomas, P. C., et al. 1997, Icarus, 128, 95

Burbine, T. H., Buchanan, P. C., Binzel, R. P., et al. 2001, Meteor. Planet. Sci., 36,761

Clayton, R. N., \& Mayeda, T. K. 1983, Earth Plan. Sci. Lett., 62, 1

Drummond, J. D., Fugate, R. Q., \& Christou, J. C. 1998, Icarus, 132, 80

Gaffey, M. J. 1997, Icarus, 127, 130

Hasegawa, S., Miyasaka, S., \& Tokimasa, N. 2009, Lunar Plan. Sci. Conf. 40th, 1503

Harris, A. W., Young, J. W., Bowell, E., et al. 1989, Icarus, 77, 171

Hendrix, A. R., Vilas, F., \& Festou, M. C. 2003, Icarus, 162, 1

Hiroi, T., Pieters, C. M., \& Takeda, H. 1994, Meteoritics, 29, 394

Keller, H. U., Barbieri, C., Lamy, P. L., et al. 2007, Space Sci. Rev., 128, 26

Küppers, M., Mottola, S., Lowry, S., et al. 2007, A\&A, 462, 13

Lagerkvist, C. I., \& Magnusson, P. 1990, A\&AS, 86, 199

Lagerkvist, C. I., Magnusson, P., Belskaya, I., et al. 1995, EAR-A-3-DDR-APCLIGHTCURVE-V1.0 NASA Planetary Data System

Li, J. Y., McFadden, L. A., Thomas, P. C., et al. 2010, Icarus, 208, 238

Li, J. Y., Thomas, P. C., Carcich, B., et al. 2011a, Icarus, 211, 528

Li, J. Y., Bodewits, D., Feaga, L. M., et al. 2011b, Icarus, submitted

McCord, T. B., Adams, J. B., \& Johnson, T. V. 1970, Science, 178, 745

McSween, H. Y. 1989, Ann. Rev. Earth Planet. Sci., 17, 119

McSween, H. Y., Mittlefehldt, D. W., Beck, A. W., Maine, R. G., \& McCoy, T. J. 2011, Space Sci. Rev., in press

Pieters, C. M., Binzel, R. P., Bogard, D., et al. 2006, Proc. IAU Symp., 229, 273 Reddy, V., Gaffey, M. J., Kelley, M. S., et al. 2010, Icarus, 210, 693

Russell, C. T., Capaccioni, F., Coradini, A., et al. 2007, Earth Moon Planets, 101, 65

Sierks, H., Keller, H. U., Jaumann, R., et al. 2011, Space Sci. Rev., in press

Thomas, P. C., Binzel, R. P., Gaffey, M. J., et al. 1997a, Icarus, 128, 88

Thomas, P. C., Binzel, R. P., Gaffey, M. J., et al. 1997b, Science, 277, 1492

Zappalá, V., Cellino, A., Barucci, A. M., Fulchignoni, M., \& Lupishko, D. F. 1990, A\&A, 231, 548

Page 5 is available in the electronic edition of the journal at http://www . aanda.org 
Table 2. Vesta lightcurve observations of the 1 May 2010.

\begin{tabular}{|c|c|c|c|c|c|}
\hline $\mathrm{UT}_{\text {start }}$ & inst. & $\exp (\mathrm{s})$ & $\Delta(\mathrm{AU})$ & $\alpha\left(^{\circ}\right)$ & $R\left(1,1,52.26^{\circ}\right)$ \\
\hline $05: 52: 12$ & NAC & 0.1546 & 0.2859060 & 52.11 & $4.455 \pm 0.005$ \\
\hline 06:24:12 & NAC & 0.1546 & 0.2857953 & 52.15 & $4.393 \pm 0.005$ \\
\hline 06:56:12 & NAC & 0.1546 & 0.2856847 & 52.18 & $4.421 \pm 0.005$ \\
\hline $07: 28: 12$ & NAC & 0.1546 & 0.2855742 & 52.22 & $4.448 \pm 0.005$ \\
\hline 08:00:11 & NAC & 0.1546 & 0.2854638 & 52.25 & $4.411 \pm 0.005$ \\
\hline 08:32:12 & NAC & 0.1546 & 0.2853535 & 52.29 & $4.472 \pm 0.005$ \\
\hline 09:04:12 & NAC & 0.1546 & 0.2852432 & 52.33 & $4.532 \pm 0.005$ \\
\hline 09:36:12 & NAC & 0.1546 & 0.2851331 & 52.36 & $4.583 \pm 0.005$ \\
\hline 10:08:12 & NAC & 0.1546 & 0.2850230 & 52.40 & 0.005 \\
\hline 10:40:12 & NAC & 0.1546 & 0.2849130 & 52 & .005 \\
\hline 10:58:25 & NAC & 0.1546 & 0.28 & 52 & .005 \\
\hline$: 13$ & NAC & 0.1 & 0.28 & & 005 \\
\hline 11:44:12 & NAC & 0.1 & 0.28 & 52 & .005 \\
\hline $12: 16: 11$ & NAC & 0.1 & 0.28 & 52 & 005 \\
\hline $12: 48: 12$ & NAC & 0.1 & 0.28 & 52 & 05 \\
\hline 13:20:11 & NAC & 0. & 0.28 & 52 & 05 \\
\hline $13: 52: 12$ & NAC & 0.1 & 0.28 & 52 & 05 \\
\hline $14: 24: 12$ & NAC & 0.1 & 0.28 & 52 & 005 \\
\hline 14:56:12 & NAC & 0.1546 & 0.2840362 & 52.73 & $4.582=$ \\
\hline $15: 28: 12$ & NAC & 0.1546 & 0.2839270 & 52.76 & $4.560=$ \\
\hline 16:00:12 & NAC & 0.1546 & 0.2838178 & 52.80 & $4.567 \pm 0.005$ \\
\hline $16: 32: 12$ & NAC & 0.1546 & 0.28 & 52.83 & $4.4 / 1 \pm$ \\
\hline :11 & WAC & 1.0078 & 0.28 & 52 & .012 \\
\hline$: 11$ & WAC & 1.0078 & 0.28 & 52 & 12 \\
\hline$: 11$ & WAC & 1.0078 & 0.28 & 52 & 12 \\
\hline & WAC & & 0.28 & & 12 \\
\hline 08:16:10 & WAC & 1.0 & 0.28 & 27 & 012 \\
\hline 11 & WAC & 1.0 & 0.2 & & 013 \\
\hline$: 11$ & $\mathrm{~W}$ & & 0.2 & & 13 \\
\hline 11 & WAC & $1 .($ & 0.28 & 52 & 13 \\
\hline 10:24:11 & WAC & 1.0 & 0.28 & 52 & 13 \\
\hline 10:56:11 & WAC & 1.0078 & 0.2848581 & 52.45 & 4.498 \\
\hline 11:09:58 & WAC & 1.0078 & 0.2848108 & 52.47 & 4.460 \\
\hline $11: 28: 12$ & WAC & 1.0078 & 0.2847482 & 52.49 & $4.424 \pm 0.012$ \\
\hline 12:00:11 & WAC & 1.0078 & 0.2846385 & 52.53 & $4.409 \pm 0.012$ \\
\hline 12:32:11 & WAC & 1.0078 & 0.2845288 & 52.56 & $4.437 \pm 0.012$ \\
\hline 13:04:11 & WAC & 1.0078 & 0.2844192 & 52.60 & $4.420 \pm 0.012$ \\
\hline 13:36:11 & WAC & 1.0078 & 0.2843097 & 52.63 & $4.418 \pm 0.012$ \\
\hline 14:08:10 & WAC & 1.0078 & 0.2842003 & 52.67 & $4.518 \pm 0.013$ \\
\hline 14:40:11 & WAC & 1.0078 & 0.2840909 & 52.71 & $4.553 \pm 0.013$ \\
\hline 15:12:11 & WAC & 1.0078 & 0.2839816 & 52.74 & $4.577 \pm 0.013$ \\
\hline 44:11 & WAC & 1.0078 & 0.2838724 & 52.78 & $4.572 \pm 0.013$ \\
\hline 16:16:11 & WAC & 1.0078 & 0.2837634 & 52.82 & $4.497 \pm 0.012$ \\
\hline 16:40:11 & WAC & 1.0078 & 0.2836816 & 52.84 & $4.446 \pm 0.013$ \\
\hline
\end{tabular}

Notes. Exp. is the exposure time, $\Delta$ is the Rosetta-Vesta distance, $\alpha$ the phase angle, and $R\left(1,1,52.26^{\circ}\right)$ the absolute magnitude reduced in the Bessel $R$ filter and corrected to a phase of $52.26^{\circ}$. 